\title{
STUDY OF INCREASE IN GLOBAL COMPONENTS IN HYBRID WAVELETS ON DATA COMPRESSION
}

\author{
Dr. H.B.Kekre ${ }^{1}$, Dr.Tanuja Sarode ${ }^{2}$ and Prachi Natu ${ }^{3}$ \\ ${ }^{1}$ Sr. Professor, MPSTME, Department of ComputerEngineering, NMIMS University, Mumbai, India \\ hbkekre@yahoo.com \\ ${ }^{2}$ Associate Professor Department of Computer Engineering, TSEC, Mumbai University, India \\ tanuja_0123@yahoo.com \\ ${ }^{3} \mathrm{Ph}$. D. Research Scholar, MPSTME, NMIMS University, Mumbai, India \\ prachi.natu@yahoo.com
}

\begin{abstract}
This paper presents a hybrid wavelet transform technique which studies the effect of global components on the quality of image compression. Hybrid wavelet transform is generated using two different component orthogonal transforms. One orthogonal transform represents global featuresof image in betterway and another is used to represent local features. Walsh transform of size $8 \times 8$ is used as a base transform i.e. to represent global characteristics of image. Other transforms like DCT, Discrete Real Fourier Transform,DiscreteHartley transform (DHT), Discrete Sine Transform (DST), Discrete Kekre Transform (DKT) and Slant transform of size 32x32 are used to focus on local characteristics of an image.256x256 hybrid wavelet transform is generated and multiple iterations of global components are included using columns of base transform and its effect on reconstructed image quality is observed in terms of Root Mean Square Error (RMSE) and Peak Signal to Noise Ratio (PSNR). From the experiments it has been observed that when DCT is used to extract local features, best results are obtained among all combinations with Walsh transform. These results are also compared with Walsh transform and observed to be much superior at higher compression ratios giving better image quality.
\end{abstract}

\section{KEYWORDS}

Hybrid Wavelet Transform, Global Components, Real Fourier Transform, Kekre Transform, DCT

\section{Council for Innovative Research}

Peer Review Research Publishing System

Journal: INTERNATIONAL JOURNAL OF COMPUTERS \& TECHNOLOGY

Vol 9, No 2

editor@cirworld.com 


\section{INTRODUCTION}

Images carryessential visual information. Increasing use of multimedia and internet has made storage and transmission of images easier. But it consumes large space, bandwidth and time to store and transfer the images. It creates an immense need to compress the images so as to reduce storage space and transmission. Eliminating redundancies in the image is the basic idea behind compression. Neighboring pixels in the image are correlated. Decorrelating these pixels removes redundancy in the image. Transforms are good mean to de-correlate pixels in the image.Many image compression techniques use transform based methods.Discrete Fourier Transform (DFT) and Short Time Fourier Transform (STFT)[1]do not give satisfactory results for nonstationary signals. DFT cannot detect local properties of a signal. STFT gives local properties at the cost of global properties.Discrete Cosine Transform(DCT) [2] is most widely used transform due to its high decorrelation property. To apply DCT, image should be divided into non overlapping blocks. It gives rise to blocking artifacts which avoids smooth degradation of image quality at the edges[3].

In recent years more attention has been given to wavelet transforms [4,5]. Wavelets provide solution to the problem of blocking artifacts as they are applied to whole image. It also gives better compression ratio due to itsbetter energy compaction property which results in better visual quality of an image. Wavelet transform reveals local characteristics of an image. So the drawback of DFT and STFT is eliminated by wavelets. Multi-resolution is another important property of wavelets. It allows viewing different frequencies at different resolutions[6,7]. Low frequencies last for entire duration of the signal whereas high frequencies appear from time to time as short burst. These properties make wavelet transforms applicable in numerous fields. The areas of application differ from science [4] to medicine and finance [5].

Next to wavelet transform comes hybrid wavelet transform. Hybrid wavelet transform is combination of two different orthogonal transforms. It combines the traits of both the component transforms. Some orthogonal transforms might give global properties in better way whereas some transforms give local characteristics in better way. Combining such two transforms to form hybrid wavelet transform gives better results. Initially only Haar wavelets had been studied. But later in recent research wavelets of Walsh[8,9,10], DCT, Kekre[11,12,13], Hartley [14] and Real DFT [15] have been proposed and studied. By changing the component orthogonal transform, hybrid wavelet transforms can be used in specific applications.

Generation of hybrid wavelet transform from two component orthogonal transform has been proposed in [16]. Two components transform matrices of size pxp and qxq are selected separately. Hybrid wavelet transform matrix of size pqxpq is generated from these component transforms. Here first ' $q$ ' number of rows of the hybrid wavelet transform matrix is calculated as the product of each element of first row of the orthogonal transform B with each of the columns of the orthogonal transform A. These rows represent global components in hybrid wavelet transform. Remaining rows are obtained by translating the rows of matrix $B$ from second row onwards.

\section{PROPOSED TECHNIQUE}

\section{Generation of Hybrid Wavelet Transform}

Consider two orthogonal transforms of size MxM and NxN respectively. Then hybrid wavelet transform of size MNxMN can be generated using these two component transforms.

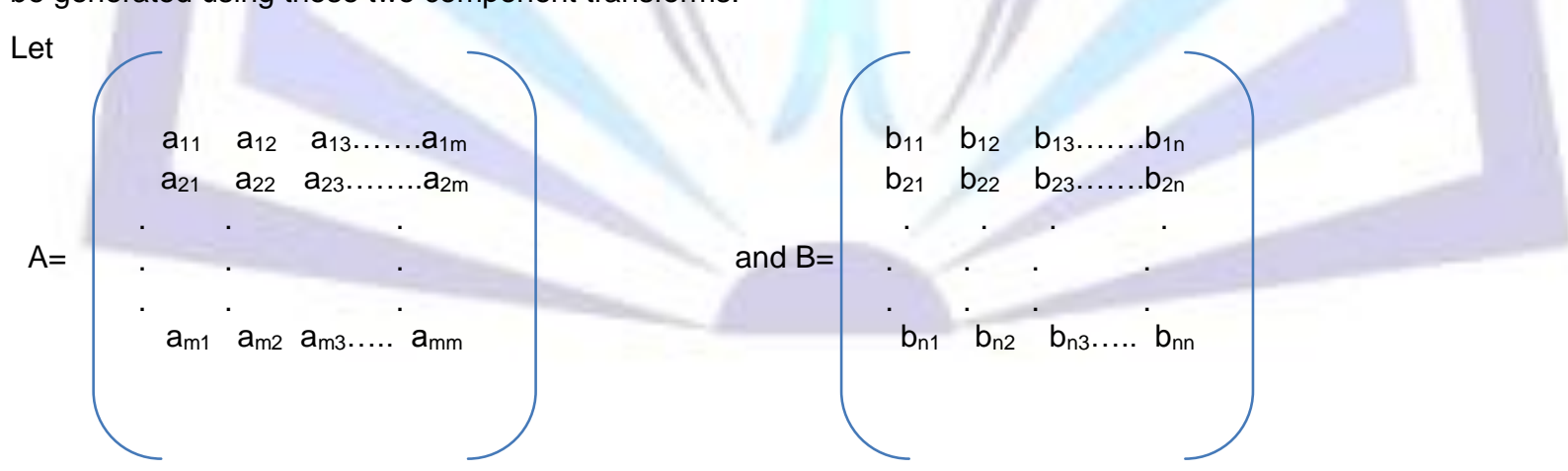

Here $A$ is of size MxM and $B$ is of size NxN then hybrid wavelet transform matrix TAB generated from $A$ and $B$ will be of size MNxMN. To obtain TAB, multiply each column of matrix $A$ by each element of first row of matrix $B$ and repeat each column of $A$, ' $N$ ' number of times. It gives first $M$ rows of TAB. To obtain next $M$ rows of matrix TAB, shift and rotate second row of matrix $B$ appending it with zeroes. Similarly all $\mathrm{N}-1$ rows of matrix $B$ are shifted and translated. In this way, in matrix TAB first $M$ rows represent global components of an image. Matrix representation of TAB is shown in Fig1. 


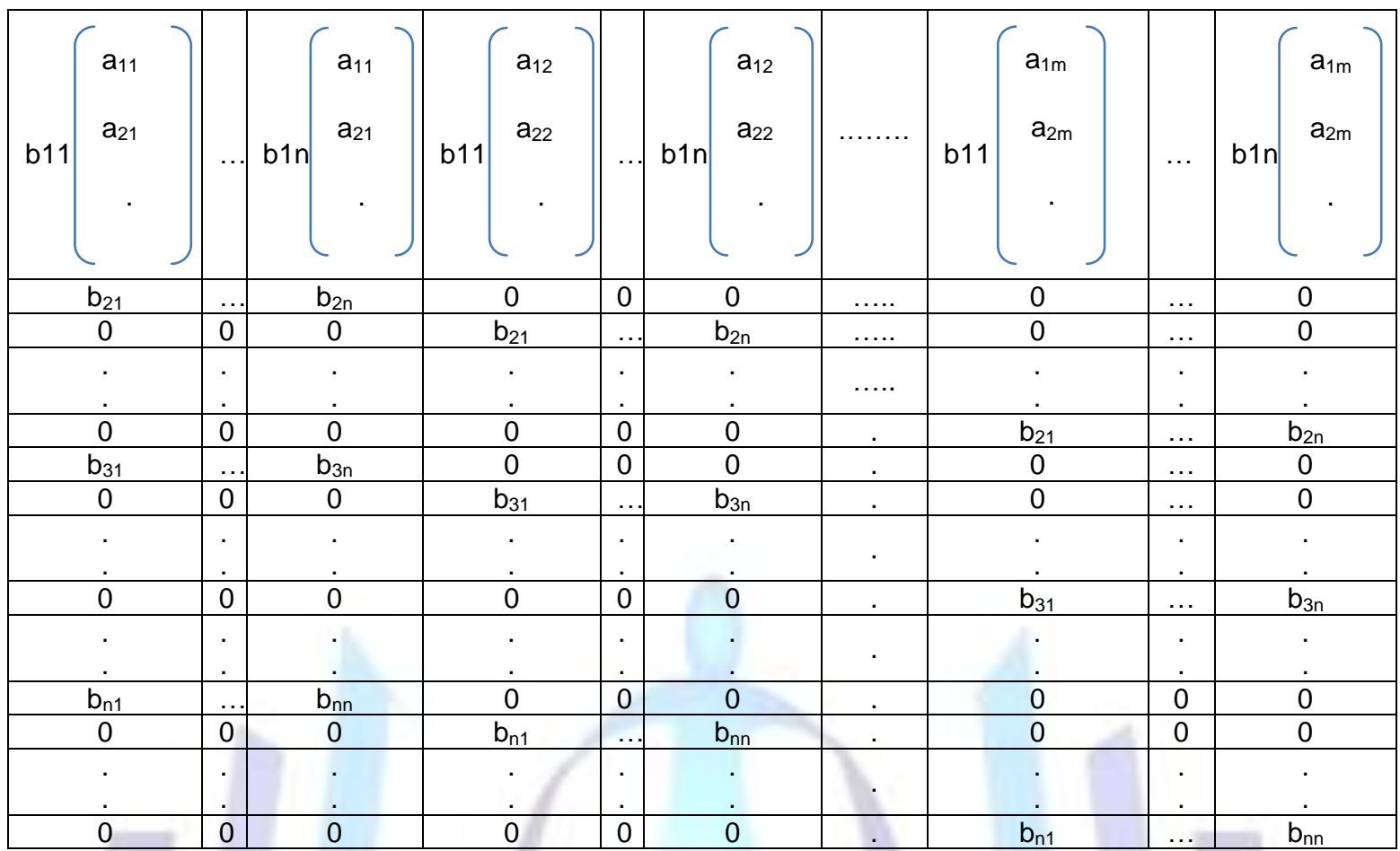

Fig 1:Matrix representation of Hybrid Wavelet Transform Matrix with first 'M' rows representing global components of an image.

To increase the contribution of global characteristics in the image, formation of $T_{A B}$ is done differently. First $M$ rows of TABare formed by repeating each column of ' $A$ ' $N$ times and multiplying each column by each element of first row of $B$. Next $M$ rows are formed by repeating each column of ' $A$ ' $N$ times and multiplying each column by each element in second row of $\mathrm{B}$.Remaining (N-2)M rows are obtained by performing shift and rotate operation on third row onwards of Matrix $B$ by appending zeroes to it.Resulting TABmatrix is shown in Fig 2. Here two iterations of column repetition of matrix $A$ are performed to increase the contribution of global components.

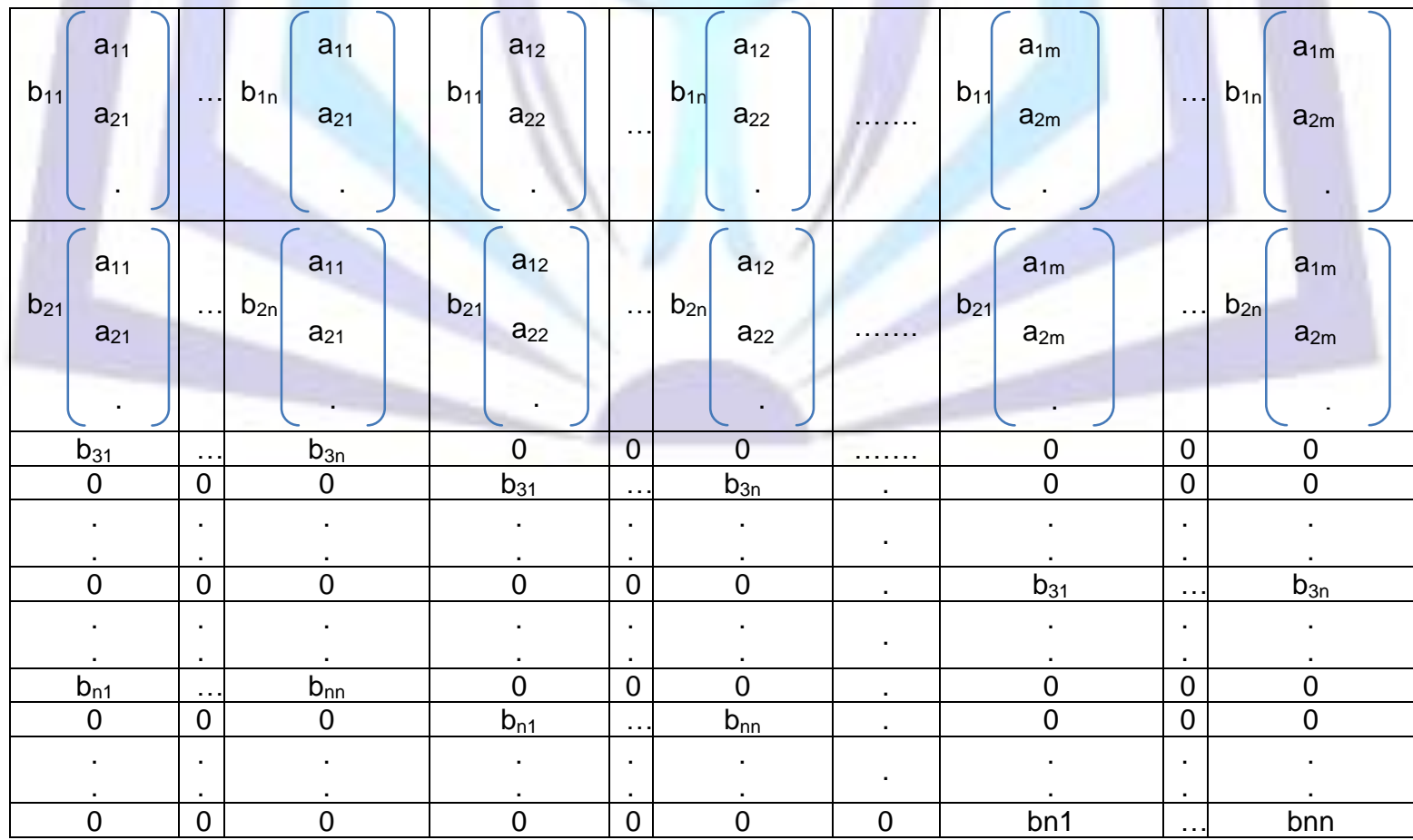

Fig2: Hybrid Wavelet Transform Matrix with first '2M' rows representing global components of image.

In matrix $T_{A B}$ we can increase contribution of global components by increasing the iterations of column repetition in matrix ' $A$ ' and multiplying each repeated column in $i^{\text {th }}$ iteration by each element in ith row of ' $B$ '. Shift and rotate operation is performed on remaining rows of matrix $\mathrm{B}$ by appending zeroes to it. When $\mathrm{N}$ iterations are performed we get kroneker 
product of $\mathrm{A}$ and $\mathrm{B}$. Thus transformation matrix to be used in image compression algorithm can be varied and results are observed in each case.

Walsh transform matrix is used to contribute global components i.e. as a base matrix. Different transforms like DCT, RealDFT, Discrete Hartley Transform (DHT), DST, Kekre Transform (DKT) and Slant are used to focus on local components of an image.

\section{Algorithm for Image Compression}

1. Consider colour image of size $256 \times 256$.

2. Separate $R, G, B$ components of an image

3. Let ' $A$ ' is Walsh transform of size $8 \times 8$ and ' $B$ ' is any of the above mentioned transforms of size $32 \times 32$, then hybrid transform of 256x256 size is generated as stated in step I of proposed technique.

4. Full Hybrid Wavelet Transform of image ' $f$ ' is obtained as ' $F$ ' $=[T A B]^{*}[f]^{\star}[T A B]^{t}$.

5. Elements in transformed plane are sorted in descending order of their energy and in each step (8*256) lowestenergy elements are eliminated.

6. Reconstruct the image by applying inverse transform.

7. Calculate RMSE and PSNR between original image and reconstructed image at different compression ratios.

8. Vary the contribution of global components and regenerate hybrid wavelet transform matrix TAB. Repeat steps 4 to 7 using this transform matrix. Compare the results in each case.

\section{EXPERIMENTS AND RESULTS}

Twelve different colour images are used for experimentation. Algorithm is run using Matlab 7.2 on AMD Dual Core processor with 4 GB RAM.
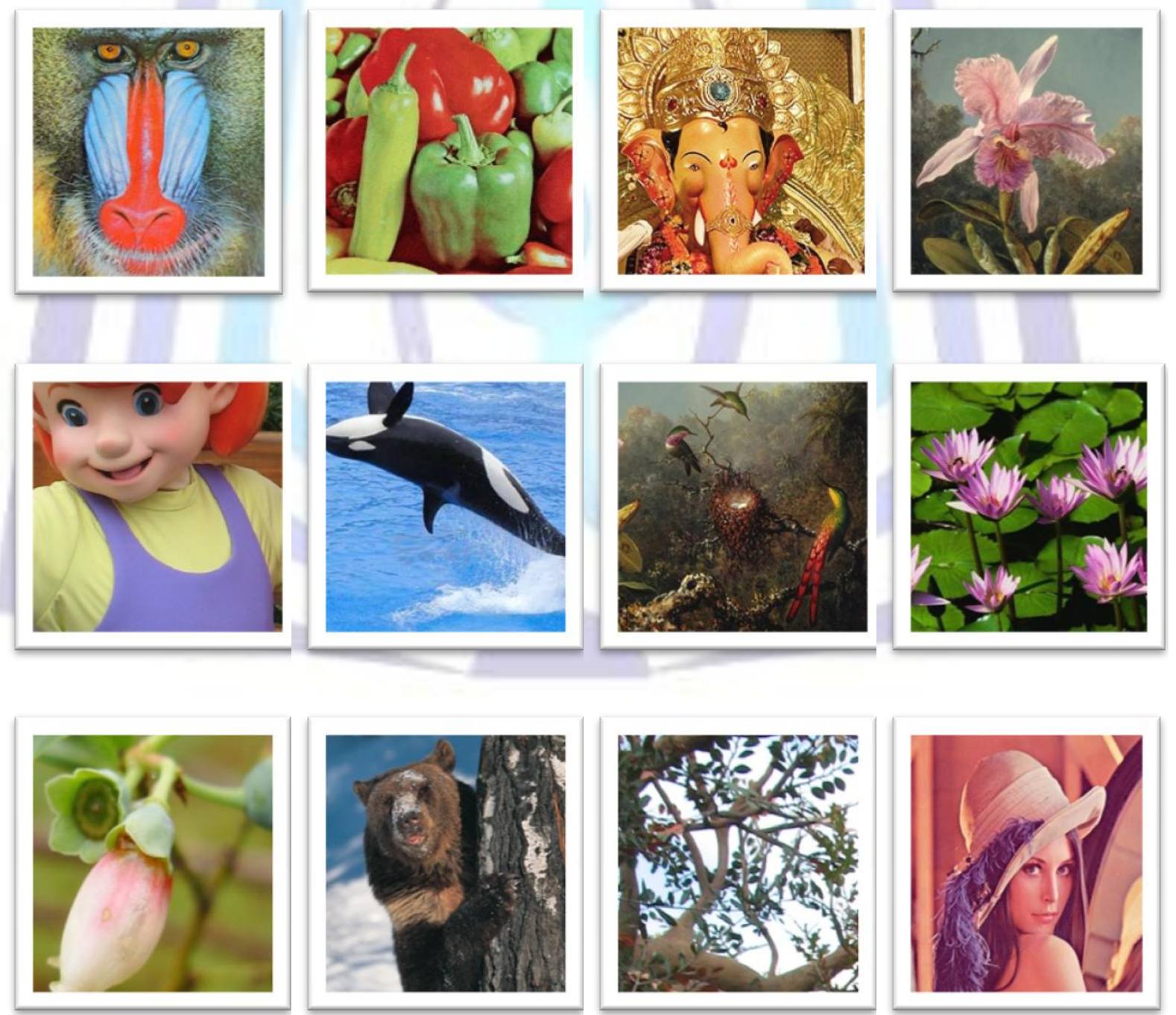

Fig3: Set of twelve test images of different classes used for experimental purpose namely (from left to right and top to bottom) Mandrill, Peppers, Lord Ganesha, Flower, Cartoon, dolphin, Birds, Waterlili, Bud, Bear, Leaves and Lenna 
Figure 4 shows average RMSE plotted against different compression ratios for DWT-DCT hybrid wavelet transform. Here DWT is selected as base transform and DCT is used to extract local features of an image.Contribution of global components is increased by varying the iterations in generation of transformation matrix as mentioned in section I of proposed technique. Number of iterations are varied from 1,2,4,8,16 and 32. As we increase the contribution of global components, less local features are extracted by second component transform. It reduces the visual quality of the image. In graph it is reflected by increase in error between original image and reconstructed image. Also with increase in compression ratio, RMSE increases.

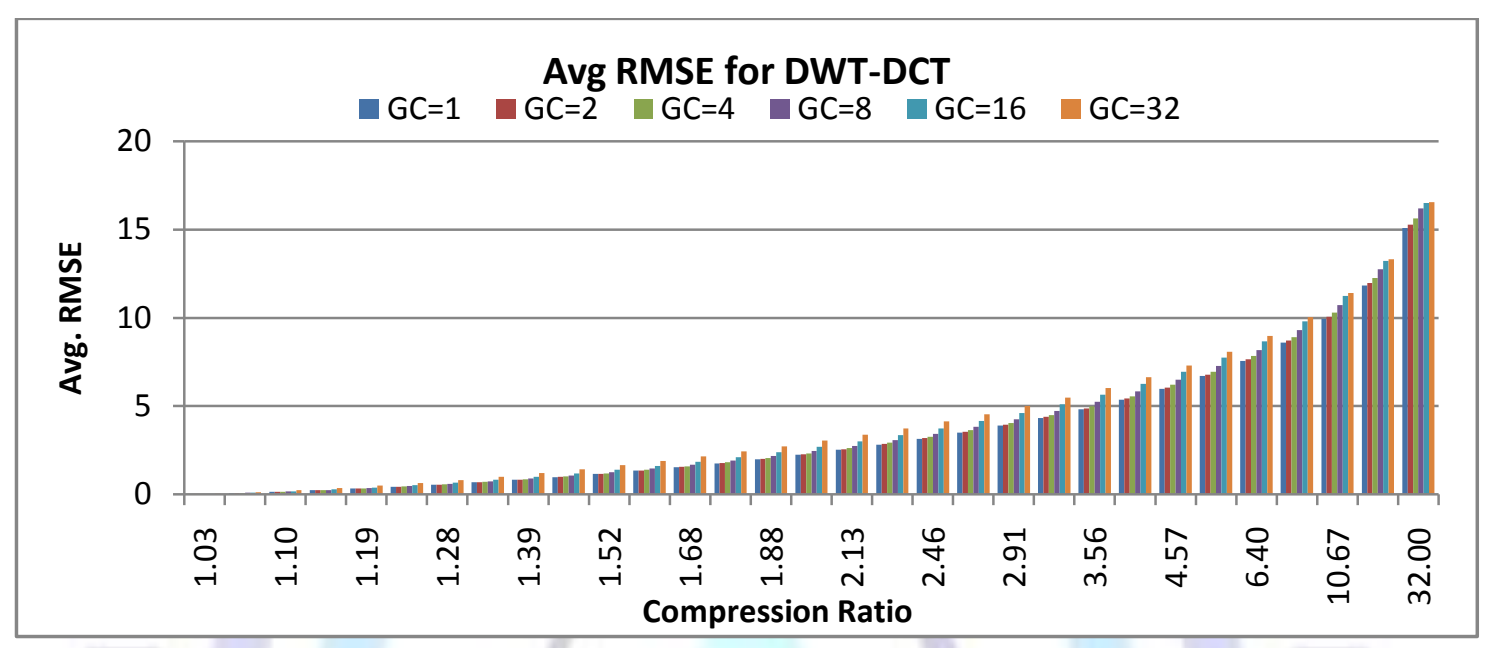

Fig4: Average RMSE vs. Compression Ratio for DWT-DCT Hybrid Wavelet with variation in Global Components

Figure 5 shows plot of Average RMSEvs. compression ratio, using Real DFT to extract the local features. With increasing focus on global components, extraction of local information reduces. It degrades quality of reconstructed image. Hence RMSE value increases as compression ratio increase.

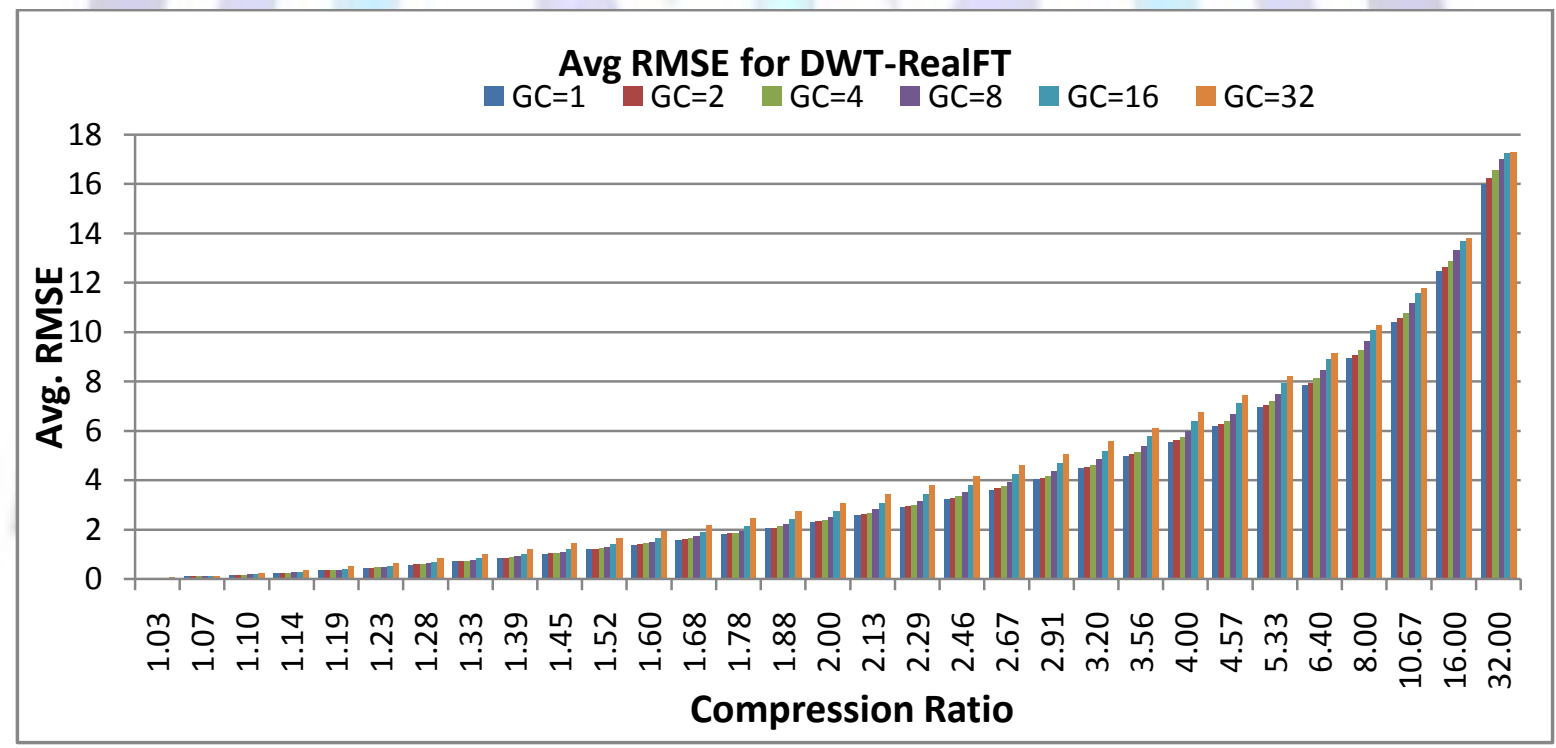

Fig5: Average RMSE vs. Compression Ratio for DWT-RealDFT Hybrid Wavelet with variation in Global Components

Figure 6 shows the graph where Discrete Hartley Transform is used to extract local properties. With increase in compression ratio and global components, RMSE value increases.

As shown in figure 7, DST is used to extract local features with Walsh transform as a base transform. From graph it can be seen that, for compression ratio greater than 8 , RMSE value decreases with increase in incorporation of global components.

Plot of Average RMSE for DWT-DKT and DWT-Slant is shown in figure 8 and 9 respectively. Minimizing the focus on local features reduces the image quality because local features are responsible for smooth representation of the image. 


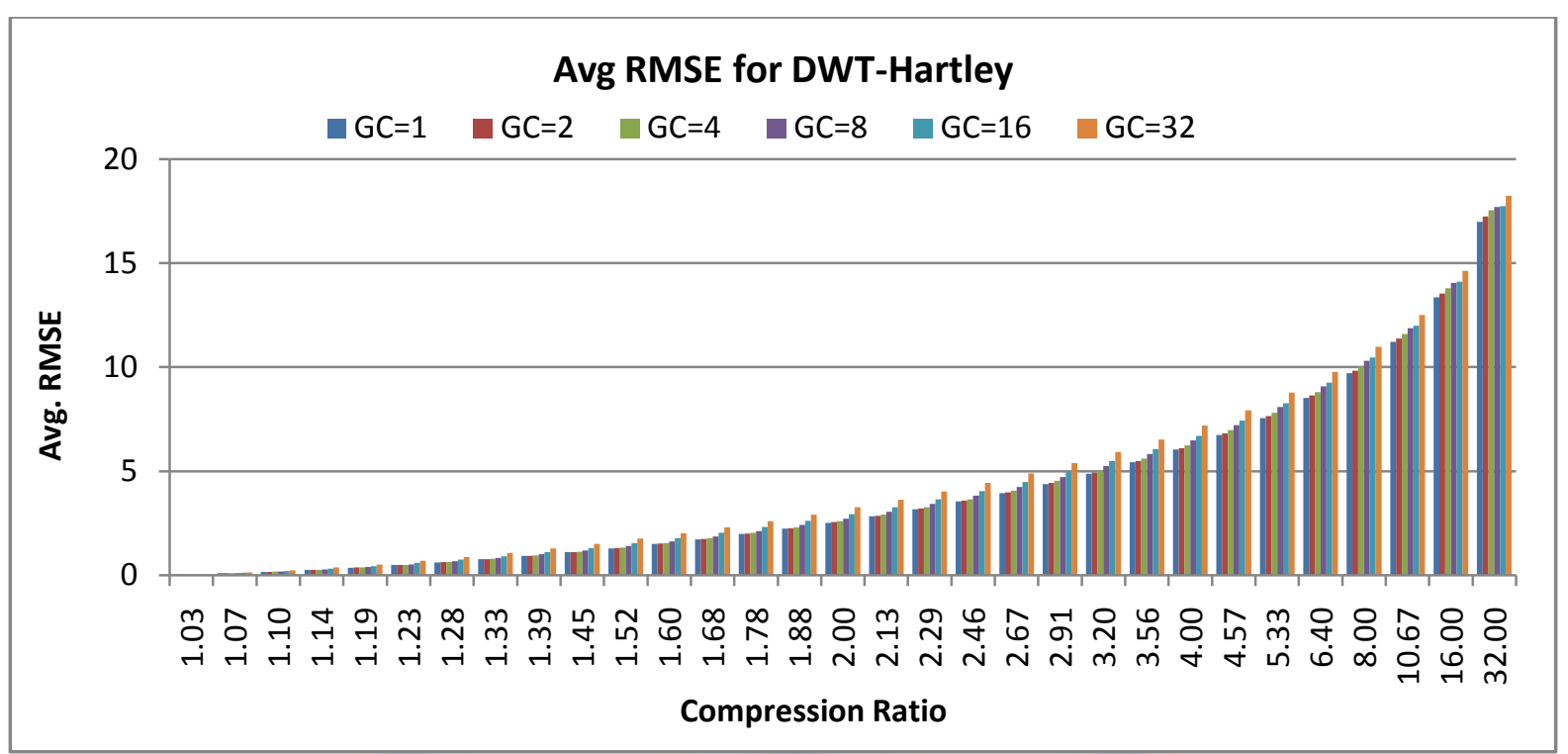

Fig 6 : Average RMSE vs. Compression Ratio for DWT-DHT Hybrid Wavelet with variation in Global Components

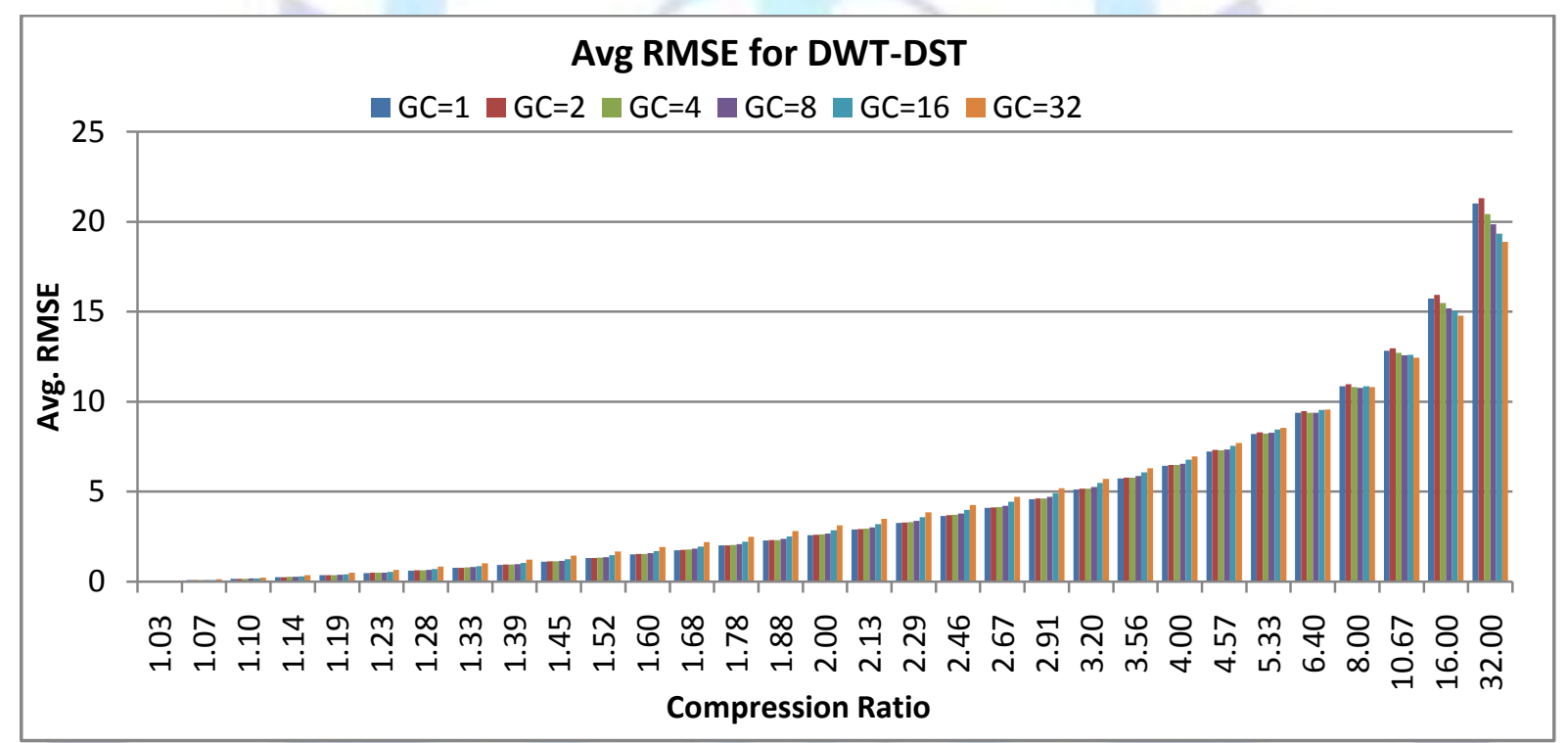

Fig 7: Average RMSE vs. Compression Ratio for DWT-DST Hybrid Wavelet with variation in Global Components

Figure 10 shows original image of 'Flower'. Six different hybrid wavelet transforms namely DWT-DCT, DWT-RealDFT, DWT-DHT, DWT-DST, DWT-DKT and DWT-Slant are applied on this image with variation in global components. Then it is reconstructed by applying inverse transform. 


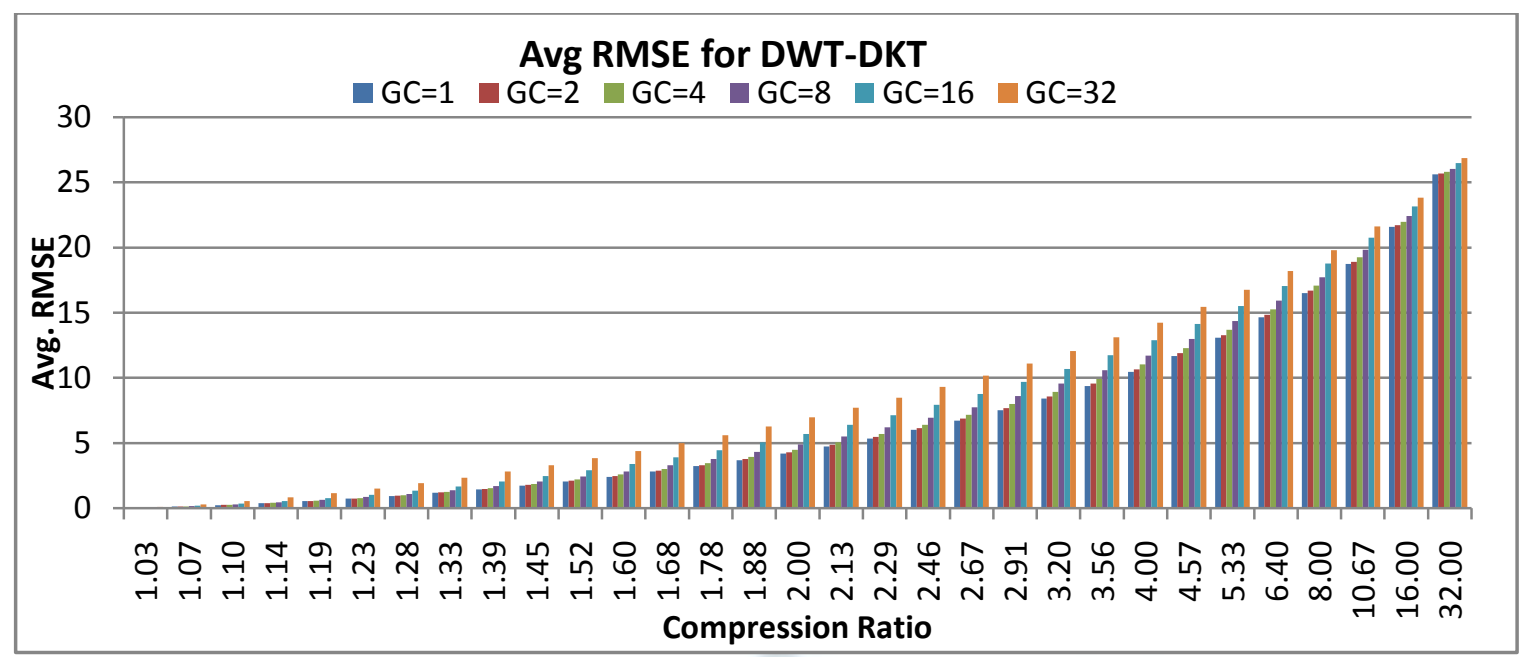

Fig 8: Average RMSE vs. Compression Ratio for DWT-DKT Hybrid Wavelet with variation in Global Components

Reconstructed images of flower at compression ratio 4 are shown in Figure 11. Similarly, figure 12 and 14 shows original image of 'Lena' and 'Peppers' and their corresponding reconstructed images at compression ratio 4 are shown in Figure 13 and 15 with respective RMSE and PSNR values. It has been observed that, RMSE values show gradual increase as global components are increased.

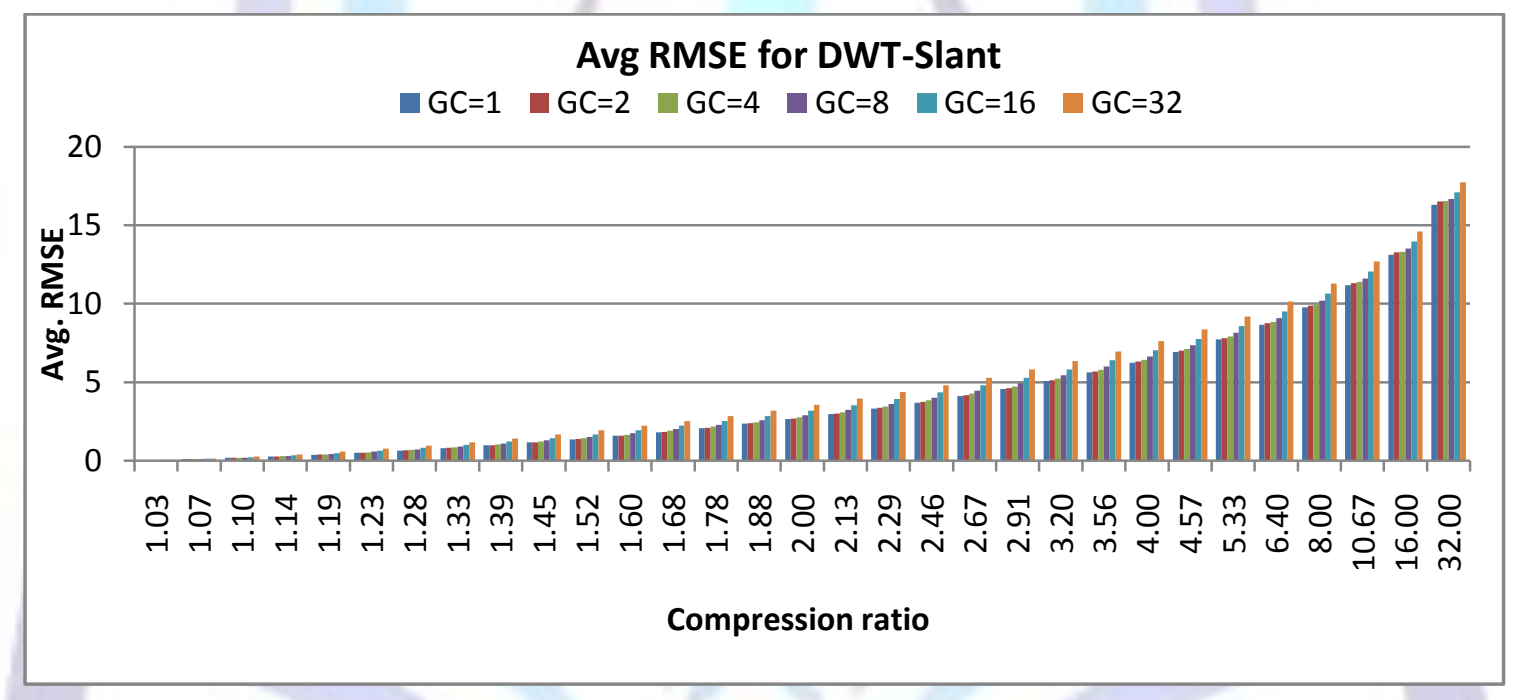

Fig 9: Average RMSE vs. Compression Ratio for DWT-Slant Hybrid Wavelet with variation in Global Components

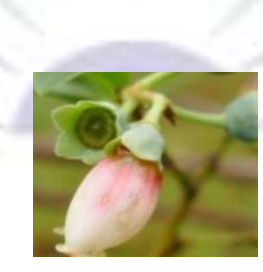

Fig10: Original Image (Flower) 


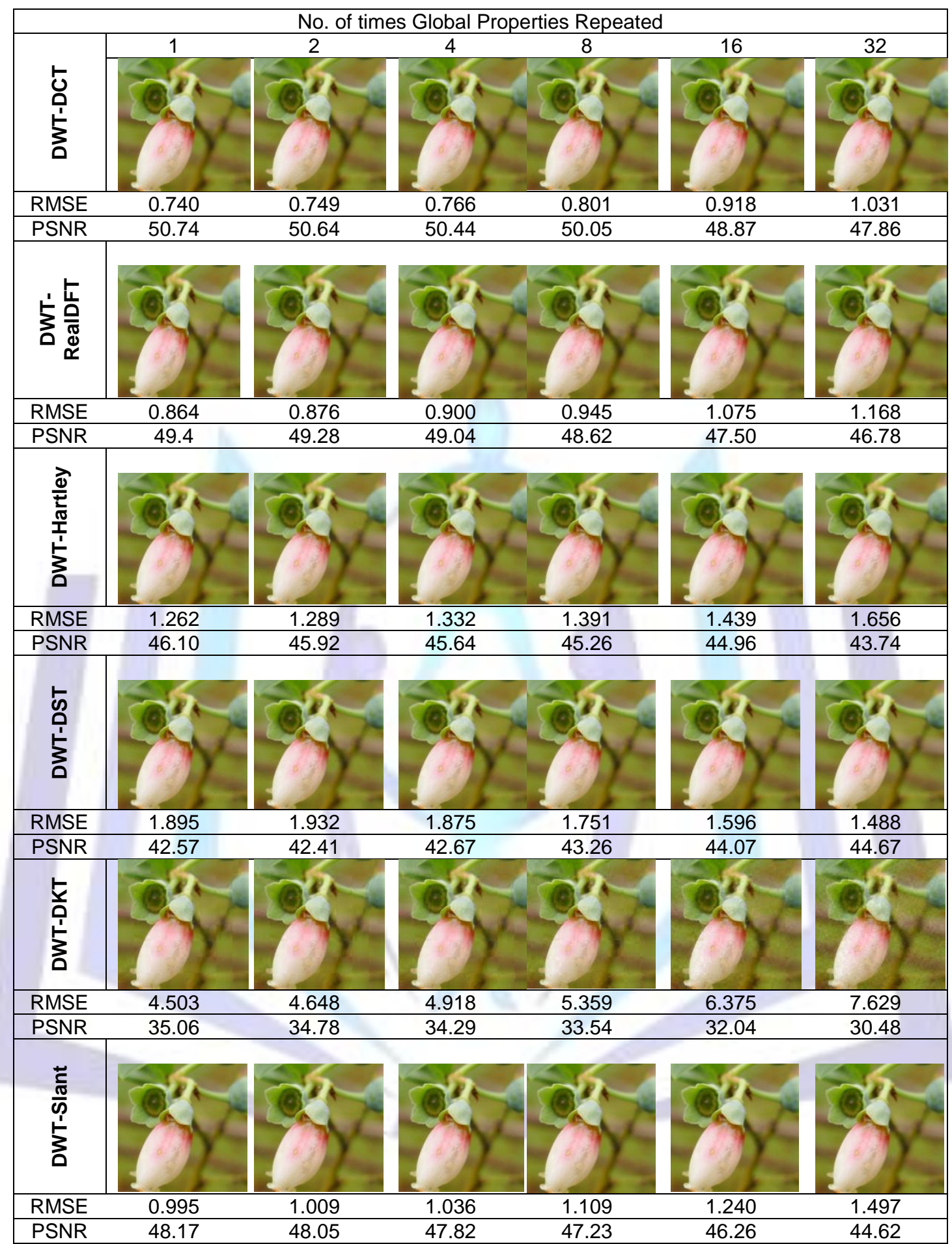

Fig11:Reconstructed 'Flower' Image using Different Hybrid Wavelet Transforms with Variation in Global Components at Compression Ratio 4 


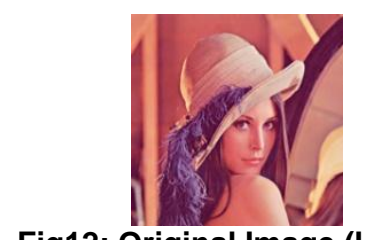

Fig12: Original Image (Lenna)

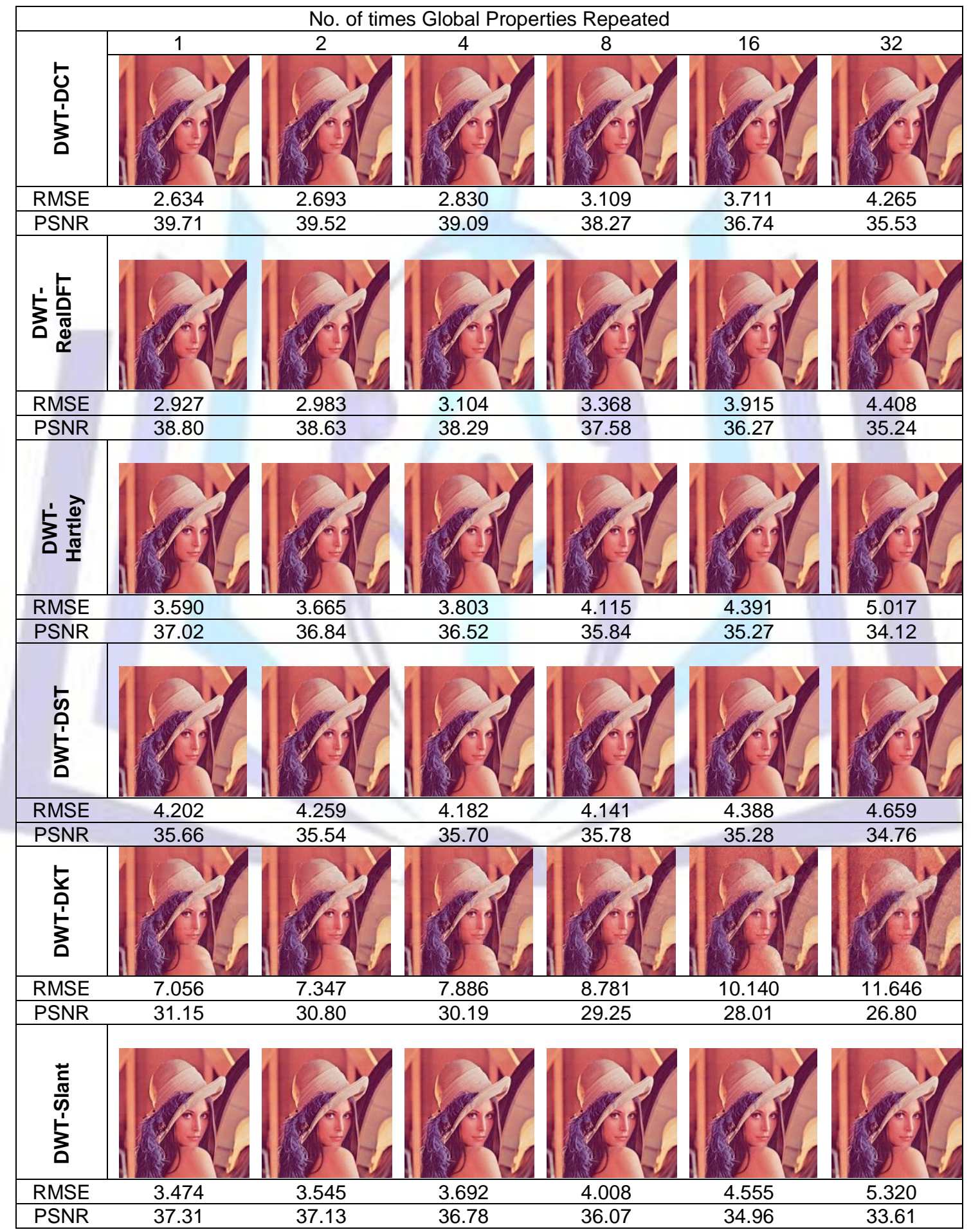

Fig 13: Reconstructed 'Lena' Image using Different Hybrid WaveletTransforms with Variation in Global Components at Compression Ratio 4 


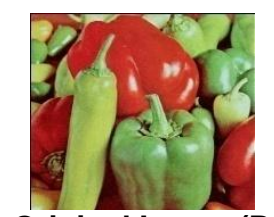

Fig 14: Original Image (Peppers)

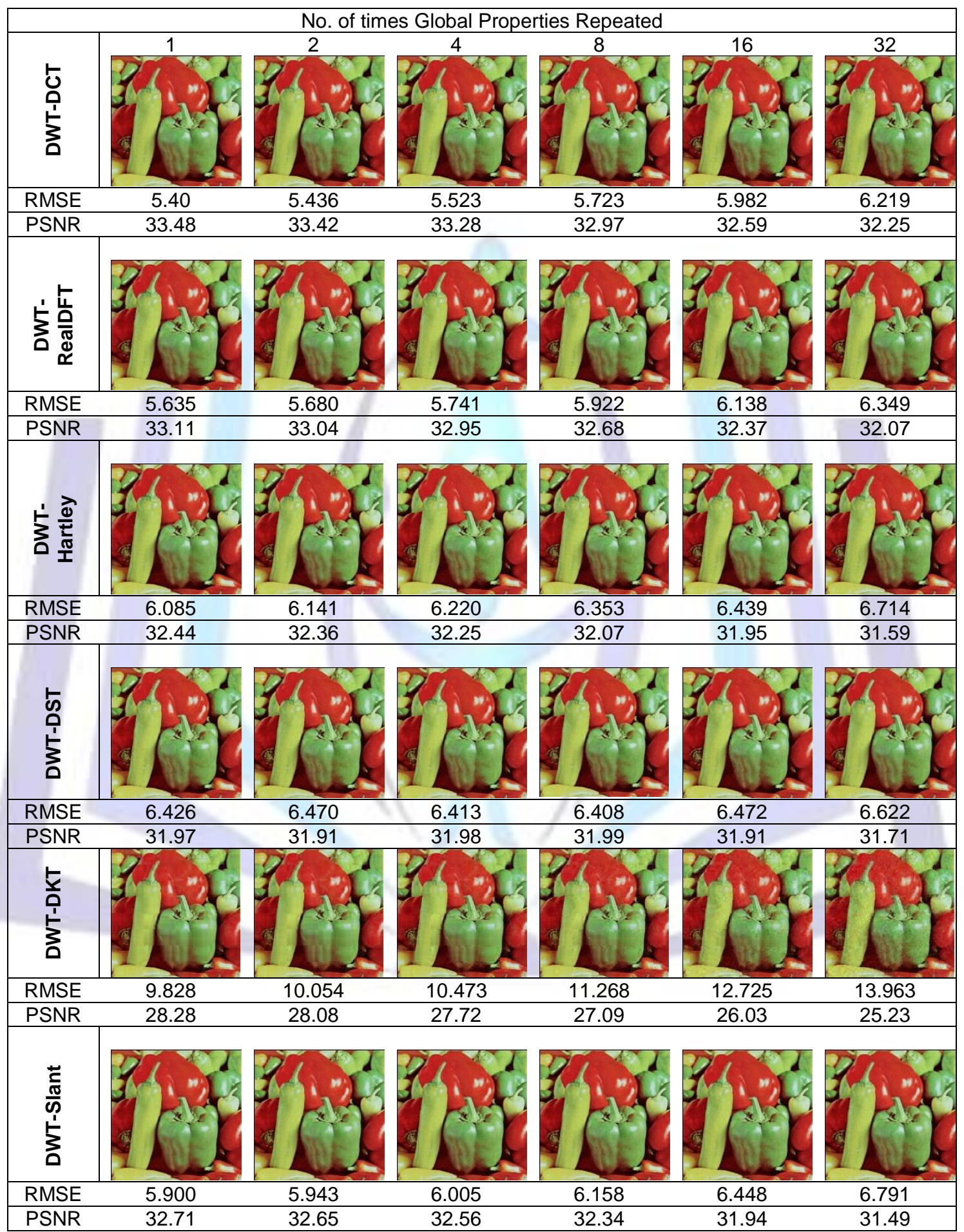

Fig 15: Reconstructed 'Peppers' Image using Different Hybrid Wavelet Transforms with Variation in Global Components at Compression Ratio 4 


\section{CONCLUSION}

This paper presents hybrid wavelet transform based image compression technique where hybrid wavelet transform matrix contributing different levels of global components can be generated. Walsh transform is used as base transform matrix and it is combined with different orthogonal component transforms that contribute to local features of an image. As more global components are focused, less local features are extracted.It introduces more distortion in the image since local features represent image smoothly. Hence RMSE valuerisesgradually and PSNR decreases. To extract local features of an image, different combinations are tried with Discrete Walsh transform (DWT). From experiments it has been observed that, DWT-DCT hybrid wavelet shows superior performance followed by DWT-RealDFT.Results of hybrid wavelet transform with variation in global components are compared with that of Walshtransform that was studied in our previous paper. Proposed method gives RMSE $0.740,5.40$ and 2.63 for 'Flower', 'Peppers' and 'Lenna' image respectively. With Walsh transform these values are $2.13,7.49$ and 6.09 respectively. It has been observed that proposed technique gives considerably better performance even at higher compression ratios. At higher compression ratio, RMSE value increasesindicating slow degradation in image quality. This degradation is more in orthogonal Walsh transform at higher compression ratio

\section{REFERENCES}

[1] Strang G. "Wavelet Transforms versus Fourier Transforms." Bull.Amer. Math. Soc. 28, 288-305, 1993.

[2] N. Ahmed, T. Natarajan and K. R. Rao, "Discrete Cosine Transform”, IEEE Transaction Computers, C-23, pp. 90-93, January 1974.

[3] Nikkoo Khalsa, Dr. G. G. Sarate, D. T. Ingole, "Factors Influencing The Image Compression Of Artificial and Natural Image Using Wavelet Transform" International Journal of Engineering Science and Technology (IJEST), Vol. 2(11), pp. 6225-6233, 2010.

[4] K. P. Soman and K.I. Ramachandran. "Insight into WAVELETS from Theory to Practice", Printice -Hall India, pp 3-7, 2005.

[5] Amara Graps, "An Introduction to Wavelets", IEEE Computational Science and Engineering, vol. 2, num. 2, Summer 1995, USA.

[6] S. Mallat, "A Theory of Multiresolution Signal Decomposition: The Wavelet Representation," IEEE Trans. Pattern Analysis and Machine Intelligence, vol. 11, pp. 674-693, 1989.

[7] M.Ashok, Dr.T.BhaskaraReddy, "Image Compression Techniques Using Modified high quality Multi wavelets", International Journal of Advanced Computer Science and Applications, IJACSA, Vol. 2, No. 7, pp.153-158, 2011.

[8] George Lazaridis, Maria Petrou, "Image Compression By Means of Walsh Transform”, IEEE Transaction on Image Processing, Volume 15, Number 8, pp.2343-2357, 2006.

[9] H.B.Kekre, Sudeep D. Thepade, Akshay Maloo, "Face Recognition using Texture Features Extracted form Walshlet Pyramid", ACEEE International Journal on Recent Trends in Engineering and Technology (IJRTET), Volume 5, Issue 1,www.searchdl.org/journal/IJRTET,2010

[10] H.B.Kekre, Sudeep D. Thepade, Juhi Jain, Naman Agrawal, "Performance Comparison of IRIS Recognition Techniques using Wavelet Pyramids of Walsh, Haar and Kekre Wavelet Transforms", International Journal of Computer Applications (IJCA), Number 2, Article 4, March 2011, http://www.ijcaonline.org/proceedings/icwet/number2/2070-aca386.

[11] H.B.kekre, Sudeep D. Thepade, Adib Parkar, "A Comparison of Haar Wavelets and Kekre's Wavelets for Storing Colour Information in a Greyscale Image", International Journal of Computer Applications (IJCA), Volume 1, Number 11, pp. 32-38,December 2010.

[12]H.B.Kekre, Sudeep D. Thepade, "Image Retrieval using Non-Involutional Orthogonal Kekre's Transform", International Journal of Multidisciplinary Research and Advances in Engineering (IJMRAE), Ascent Publication House, 2009, Volume 1, No.I, pp. 189-203, 2009. Abstract available online at www.ascentjournals.com

[13] H.B.Kekre, Sudeep D. Thepade, Archana Athawale, Anant S.Prathamesh V. Suraj S., "Kekre Transform over Row Mean, Column Mean and Both using Image Tiling for Image Retrieval", International Journal of Computer and Electrical Engineering (IJCEE), Volume 2, Number 6, October 2010, pp 964-971, is available at www.ijcee.org/papers/260-E272.pdf

[14] R. N. Bracewell, "Discrete Hartley transform," Journal of Opt. Soc.America, Volume 73, Number 12, pp. 1832-183, 1983.

[15] H.B.Kekre, Tanuja Sarode, Prachi Natu, "Image Compression using Real Fourier Transform, It's Wavelet Transform and Hybrid Wavelet with DCT", Accepted in International Journal of Advanced Computer Science and Applications,(IJACSA) Vol. 4, No.5, pp.41-47,2013. 
[16] H.B. Kekre, Tanuja Sarode, Sudeep Thepade, "Inception of Hybrid Wavelet Transform using Two Orthogonal Transforms and It's use For Image Compression", International Journal of Computer Science and Information Security(IJCSIS),Vol. 9, No. 6, pp. 80-87,2011

\section{AUTHORS PROFILE}

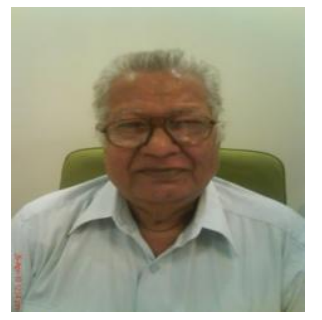

Dr. H. B. Kekre has received B.E (Hons.) in Telecomm Engineering from Jabalpur University in 1958, M.Tech (Industrial Electronics) from IIT Bombay in 1960, M.S.Engg. (Electrical Engg.) from University of Ottawa, Canada in 1965 and Ph.D. (System Identification) from IIT Bombayin 1970. He has worked as Faculty of Electrical Engg. and then HOD Computer Science and Engg. at IIT Bombay. After serving IIT for 35 years he retired in 1995. After retirement from IIT, for 13 years he was working as a professor and head in the Department of Computer Engg. and Vice Principal at Thadomal Shahani Engineering. College, Mumbai. Now he is Senior Professor at MPSTME, SVKM's NMIMS University.

He has guided 17 Ph.Ds, more than 100 M.E./M.Tech and several B.E./ B.Tech projects, while in IIT and TSEC. His areas of interest are Digital Signal processing, Image Processing and Computer Networking. He has more than 450 papers in National / International Journals and Conferences to his credit. He was Senior Member of IEEE. Presently He is Fellow of IETE, Life Member of ISTE and Senior Member of International Association of Computer Science and Information Technology (IACSIT). Recently fifteen students working under his guidance have received best paper awards. Currently eight research scholars working under his guidance have been awarded Ph. D. by NMIMS (Deemed to be University). At present eight research scholars are pursuing Ph.D. program under his guidance.

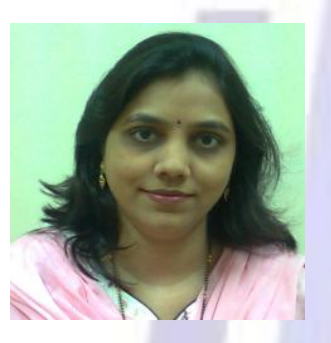

Dr. Tanuja K. Sarode has received M.E. (Computer Engineering) degree from Mumbai University in 2004, Ph.D. from Mukesh Patel School of Technology, Management and Engg. SVKM's NMIMS University, Vile-Parle (W), Mumbai, INDIA. She has more than 11 years of experience in teaching. Currently working as Assistant Professor in Dept. of Computer Engineering at Thadomal Shahani Engineering College, Mumbai. She is member of International Association of Engineers (IAENG) and International Association of Computer Science and Information Technology (IACSIT). Her areas of interest are Image Processing, Signal Processing and Computer Graphics. She has 150 papers in National /International Conferences/journal to her credit.

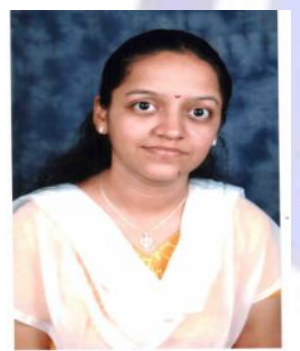

Ms.Prachi Natu has received B.E. (Electronics and Telecommunication) degree from Mumbai University in 2004. Currently pursuing Ph.D. from NMIMS University. She has 08 years of experience in teaching. Currently working as Assistant Professor in Department of Computer Engineering at Mukesh Patel School of Technology, Management and Engineering. Vile Parle (Mumbai). Her areas of interest are Image Processing, Database Management Systems and Operating Systems. She has 16 papers in International Conferences/journal to her credit. 